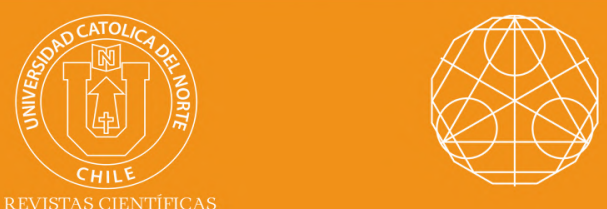

\title{
Pebbling on zig-zag chain graph of $n$ odd cycles
}

\author{
A. Lourdusamy* iD orcid.org/0000-0001-5961-358X \\ J. Steffi ${ }^{* *}$
}

*St. Xavier's College (Autonomous), Dept. of Mathematics, Palayamkottai, TN, India.

$\checkmark$ lourdusamy15@gmail.com

${ }^{* *}$ St. Xavier's College (Autonomous), Dept. of Mathematics, Palayamkottai, TN, India.

$\checkmark$ jenifersteffi.j@gmail.com

\section{Abstract:}

Given a distribution of pebbles on the vertices of a connected graph $G$, a pebbling move on $G$ consists of removing two pebbles from one vertex and placing one pebble on an adjacent vertex. The pebbling number of $G, f(G)$, is the least $n$ such that any distri-

Keywords: Graph pebbling; Zig-zag chain graph.

\section{Cite this article as (IEEE citation style):}

A. Lourdusamy and J. Steffi, "Pebbling on zig-zag chain graph of $\mathrm{n}$ odd cycles", Proyecciones (Antofagasta, On line), vol. 38, no. 3, pp. 597-615, August 2019, doi: 10.22199/issn.0717-6279-2019-030038 [Accessed dd-mm-yyyy].

Article copyright: (C) 2019 Arockiam Lourdusamy and J. Jenifer Steffi. This is an open access article distributed under the terms of the Creative Commons Licence, which permits unrestricted use and distribution provided the original author and source are credited. 


\section{Introduction}

In this work it is considered undirected simple graphs that are connected. For some other graph theoretical concepts the reader should refer to [1]. Throughout the text the letter $v$ denotes a specified vertex of the underlying graph.

Pebbling in graph was first suggested by Lagarias and Saks and it was introduced in the literature by Chung [2]. She studied the pebbling number of Cartesian product of paths to solve the following number theoretical conjecture by Klietman and Lemke [3].

Conjecture 1.1. For any given integers $a_{1}, a_{2}, \ldots, a_{d}$ there is a non-empty subset $X \subseteq\{1,2, \ldots, d\}$ such that $d$ divides $\sum_{i \in X} a_{i}$ and $\sum_{i \in X} \operatorname{gcd}\left(a_{i}, d\right) \leq$ $d$.

Graph pebbling problems are computationally complex mathematical problems. Oversimplifying things a bit, computers can only solve graph pebbling puzzles by using brute force methods, looking at every possible configuration. Graph pebbling problems belong to a large class of problems called NP-Complete. Put simply, these are problems whose best known algorithms require an exponential amount of time to solve. Specifically, the subject has grown into a network optimization model for the transportation of consumable resources. It analyzes whether it is possible to move resources from one node to another by using minimum resources in the transporting process. Thus it is very fluid and adaptable to various situations. Related pebbling models have found applications in computational complexity, compiler theory, graph searching, sparse matrix factorization, and computational geometry. To learn more about graph pebbling and its applications, readers are directed to refer to [7] and [8].

Moreover, this can be viewed as an adversarial process: You are given a graph and a set of pebbles to place wherever you wish on the graph. Your adversary will choose any node on the graph he wishes. You then have to move a pebble onto that chosen node. You will win if you can place the pebbles such that it is possible to reach every node.

Formally, the theory of graph pebbling is moving one pebble to a destined, but an arbitrary vertex $v$, for a given distribution of pebbles on the vertices of $G$ and also compute how many pebbles are necessary to guarantee that, from any configuration of that many pebbles, one can move a 
pebble to (solve) any particular vertex. Let $D$ be a distribution of pebbles on the vertices of $G$. Let $p(v)$ denote the number of pebbles distributed on the vertex $v$ and let $p(G)=\sum_{v \in V(G)} p(v)$. The operation of pebbling movement is called a pebbling step, defined as removing two pebbles from a vertex and adding one on an adjacent vertex. The following definitions will be introduced in order to understand the pebbling concepts.

Definition 1.2. $[2,6]$ We define the t-pebbling number of $v$ in $G$ is the smallest number $f_{t}(G, v)$ such that from every placement of $f_{t}(G, v)$ pebbles, it is possible to move $t$ pebbles to $v$. For $t=1$, we have the pebbling number of a vertex $v$ in $G$ denoted by $f(G, v)$ such that, it is possible to move a pebble to $v$ by a sequence of pebbling moves.

Definition 1.3. $[2,6]$ The t-pebbling number of $G$ is the smallest number, $f_{t}(G)$, such that from any placement of $f_{t}(G)$ pebbles, it is possible to move $t$ pebbles to any specified, but an arbitrary vertex, by a sequence of pebbling moves. Thus, $f_{t}(G)$ is the maximum value of $f_{t}(G, v)$ over all vertices $v$. For $t=1$, we can get the pebbling number of $G$, denoted by $f(G)$. It is the maximum value of $f(G, v)$ over all vertices $v$. Thus we can move a pebble to any arbitrary vertex by a sequence of pebbling moves.

With regard to pebbling number and $t$-pebbling number of various classes of graphs, we invite the readers to refer to $[2,4,9,10]$, and [11]. Motivated by these works, we compute the pebbling number and the $t$-pebbling number of zig-zag chain graph of $n$ copies of odd cycles. This paper is organized as follows. In Section 2, we define the zig-zag chain graph of $n$ copies of odd cycles, $Z Z_{n}\left(C_{2 k+1}\right)$, and present some important lemmas to prove the main results. In Section 3, we provide the pebbling number of $Z Z_{n}\left(C_{2 k+1}\right)$, for some odd cycles $C_{2 k+1}, 1<k<6$ and in Section 4, we determine the $t$-pebbling number for the same.

\section{Preliminaries}

In this section, we provide some important facts and set up notations and terminologies. Initially, we proceed with the study of zig-zag stucture which can be found in study of nano tube related concepts in chemistry for hexagon type. We have noted that hexagon cycle is included in studying zig-zag chain graph of $n$ copies of even cycles [14]. Here, we focus on the zig-zag chain graph of $n$ copies of odd cycles. In [15], the authors proved that this graph satisfied the 2t-pebbling property. In this paper, we are going to compute the $t$-pebbling number for the same. 
Definition 2.1. [15] The zig-zag chain graph of $n$ copies of odd cycles denoted by $Z Z_{n}\left(C_{2 k+1}\right)$, is a graph which consists of zig-zag sequence of $n$ copies of odd cycles, $C_{2 k+1}$ with $k \geq 2$. We define $Z Z_{n}\left(C_{2 k+1}\right)$ as follows.

Case $(i)$ When $n$ is even.

The vertex set of $Z Z_{n}\left(C_{2 k+1}\right)$ is

$$
V\left(Z Z_{n}\left(C_{2 k+1}\right)\right)=\left\{a_{i}, b_{i}: 1 \leq i \leq \frac{n}{2}(2 k-1)\right\} \cup\{x, y\}
$$

The edge set of $Z Z_{n}\left(C_{2 k+1}\right)$ is

$$
\begin{gathered}
E\left(Z Z_{n}\left(C_{2 k+1}\right)\right)=\left\{a_{i} a_{i+1}, b_{i} b_{i+1}: 1 \leq i \leq \frac{n}{2}(2 k-1)-1\right\} \cup \\
\left\{x a_{1}, x b_{1}, y a_{\frac{n}{2}(2 k-1)}, y b_{\frac{n}{2}(2 k-1)}\right\} \cup \\
\left\{a_{i(2 k-1)-(k-1)} b_{i(2 k-1)-(k-1)}, a_{j(2 k-1)} b_{j(2 k-1)+1}:\right. \\
\left.1 \leq i \leq \frac{n}{2}, 1 \leq j \leq \frac{n-2}{2}\right\} .
\end{gathered}
$$

Case (ii) When $n$ is odd.

The vertex set of $Z Z_{n}\left(C_{2 k+1}\right)$ is

$$
V\left(Z Z_{n}\left(C_{2 k+1}\right)\right)=\left\{a_{i}, b_{i}: 1 \leq i \leq\left(n k-\frac{n+1}{2}\right)\right\} \cup\{x, y, z\}
$$

The edge set of $Z Z_{n}\left(C_{2 k+1}\right)$ is

$$
\begin{aligned}
& E\left(Z Z_{n}\left(C_{2 k+1}\right)\right)=\left\{a_{i} a_{i+1}, b_{i} b_{i+1}: 1 \leq i \leq\left(n k-\frac{n+1}{2}\right)-1\right\} \cup \\
& \left\{x a_{1}, x b_{1}, y a_{\left(n k-\frac{n+1}{2}\right)}, y z, z b_{\left(n k-\frac{n+1}{2}\right)}\right\} \cup \\
& \left\{a_{i(2 k-1)-(k-1)} b_{i(2 k-1)-(k-1)}, a_{j(2 k-1)} b_{j(2 k-1)+1}: 1 \leq i, j \leq \frac{n-1}{2}\right\} .
\end{aligned}
$$


The Figure 1.1 depicts the zig-zag chain graph of $n$ copies of $C_{5}$.

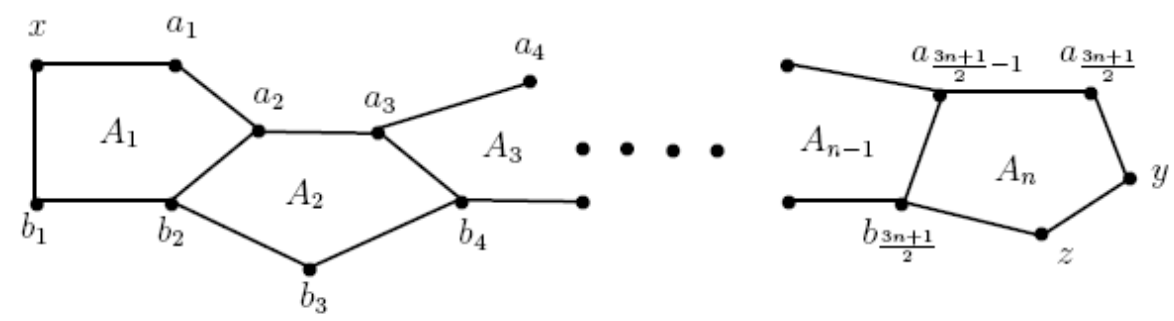

Figure 1.1

The reader can easily view that the above graph $Z Z_{n}\left(C_{5}\right)$ has $n$ copies of $C_{5}$, and we label each cycle as $A_{1}, A_{2}, \ldots$, and $A_{n}$ in order from left to right. Let $S$ be any subgraph of $Z Z_{n}\left(C_{2 k+1}\right)$ and let $\left\langle Z Z_{n}\left(C_{2 k+1}\right)-S\right\rangle$ be the induced subgraph of the graph $Z Z_{n}\left(C_{2 k+1}\right)$, obtained by removing a vertex set of the subgraph $S$ from the vertex set of the graph $Z Z_{n}\left(C_{2 k+1}\right)$. We state below some results on graph pebbling which will be used in our discussion.

Lemma 2.2. [2] The pebbling number of a graph $G$ satisfies,

$f(G) \geq \max \left\{2^{\operatorname{diam}(G)},|V(G)|\right\}$, where $\operatorname{diam}(G)$ is the diameter of the graph $G$.

Lemma 2.3. [11] Let $P_{n}$ be the path with $n$ vertices. Then

$$
f_{t}\left(P_{n}\right)=t 2^{n-1} \text {. }
$$

Lemma 2.4. [11] Let $C_{n}$ denote a simple cycle with $n$ vertices, where $n \geq 3$. Then

$$
f_{t}\left(C_{n}\right)= \begin{cases}t 2^{k}, & n=2 k \\ \frac{2^{k+2}-(-1)^{k+2}}{3}+(t-1) 2^{k}, & n=2 k+1 .\end{cases}
$$

Theorem 2.5. [14] In a zig-zag chain graph of $n$ copies of even cycles $C_{2 k}$, denoted by $Z Z_{n}\left(C_{2 k}\right)$ with a specified vertex $v$, the following are true for $k \geq 3$. 
1. If $2^{n(k-1)+1}$ pebbles are assigned to the vertices of the graph $Z Z_{n}\left(C_{2 k}\right)$, one pebble can be moved to $v$.

2. Let $q$ be the number of vertices with at least one pebble. If there are all together more than $2\left(2^{n(k-1)+1}\right)-q$ pebbles, then two pebbles can be moved to $v$.

Lemma 2.6. Let $Z Z_{2}\left(C_{2 k+1}\right)$ be the zig-zag chain graph of two copies of odd cycles. If more than $2^{2 k}-\left(\frac{2^{k+2}-(-1)^{k+2}}{3}\right)$ pebbles are distributed only on the vertices of $A_{1}$, then we can move at least $2^{k-1}$ pebbles to the vertex $a_{k}$.

Proof. Consider the graph $Z Z_{2}\left(C_{2 k+1}\right)$ with at least $2^{2 k}-\left(\frac{2^{k+2}-(-1)^{k+2}}{3}\right)+$ 1 pebbles distributed only on the vertices of $A_{1}$. We have to move at least $2^{k-1}$ pebbles to the vertex $a_{k}$. Recall that $f_{t}\left(C_{2 k+1}\right) \geq\left(\frac{2^{k+2}-(-1)^{k+2}}{3}\right)+$ $2^{k}(t-1)$. We have the following claim to prove Lemma 2.6 .

$\operatorname{Claim}(1) .2^{2 k}-\left(\frac{2^{k+2}-(-1)^{k+2}}{3}\right)+1 \geq\left(\frac{2^{k+2}-(-1)^{k+2}}{3}\right)+2^{k}\left(2^{k-1}-1\right)$.

We have,

$$
\begin{aligned}
2^{2 k}-2\left(\frac{2^{k+2}-(-1)^{k+2}}{3}\right)-2^{k}\left(2^{k-1}\right. & -1)+1>2^{k}\left(2^{k-1}+1\right)-2\left(\frac{2^{k+2}-(-1)^{k+2}}{3}\right) \\
& =\frac{1}{3}\left[3.2^{k}\left(2^{k-1}+1\right)-2\left(2^{k+2}-(-1)^{k+2}\right)\right] \\
& \geq \frac{1}{3}\left[3.2^{k}\left(2^{k-1}+1\right)-2^{k+3}-2\right] \\
& >\frac{2}{3}\left(2^{k-1}-1\right), \text { since } k \geq 2 \\
& \geq 0 .
\end{aligned}
$$

Therefore, we can move $2^{k-1}$ pebbles to the vertex $a_{k}$, which completes the proof of the lemma.

Lemma 2.7. Let $Z Z_{3}\left(C_{2 k+1}\right)$ be the zig-zag chain graph of three copies of odd cycles. If more than $2^{3 k-1}+3 k-2-\left(\frac{2^{k+2}-(-1)^{k+2}}{3}\right)$ pebbles are distributed on the vertices of $A_{1} \cup A_{2}$, then we can move at least $2^{k-1}$ pebbles to the vertex $a_{2 k-1}$. 
Proof. Consider the graph $Z Z_{3}\left(C_{2 k+1}\right)$ with more than $2^{3 k-1}+3 k-2-$ $\left(\frac{2^{k+2}-(-1)^{k+2}}{3}\right)$ pebbles distributed only on the vertices of $A_{1} \cup A_{2}$. We have to move at least $2^{k-1}$ pebbles to the vertex $a_{2 k-1}$. Suppose that more than $2^{2 k}-\left(\frac{2^{k+2}-(-1)^{k+2}}{3}\right)$ pebbles are distributed on the vertices of $A_{2}$. Then by Lemma 2.6, we can move at least $2^{k-1}$ pebbles to the vertex $a_{2 k-1}$. Therefore, assume that at most $2^{2 k}-\left(\frac{2^{k+2}-(-1)^{k+2}}{3}\right)-1$ pebbles are distributed on $A_{2}$. Then at least $2^{3 k-1}+3 k-2^{k}-1$ pebbles are retained on the vertices of $A_{1}$. We claim the following:

$\operatorname{Claim}(2) .2^{3 k-1}+3 k-2^{2 k}-1 \geq\left(\frac{2^{k+2}-(-1)^{k+2}}{3}\right)+2^{k}\left(2^{2 k-2}-1\right)$.

We have,

$$
\begin{aligned}
2^{3 k-1}-2^{2 k}+ & 3 k-1-\left(\frac{2^{k+2}-(-1)^{k+2}}{3}\right)-2^{k}\left(2^{2 k-2}-1\right) \\
& >2^{3 k-2}-2^{k}\left(2^{k}-1\right)+3 k-2^{k+1}-1, \text { since }\left(\frac{2^{k+2}-(-1)^{k+2}}{3}\right)<2^{k+1} \\
& =2^{k-2}\left(2^{2 k}-2^{k+2}-4\right)+3 k-1 \\
& \geq 2^{2 k}-2^{k+2}+3 k-5 \\
& \geq 3 k-5 \\
& >0, \text { since } k \geq 2 .
\end{aligned}
$$

Hence $p\left(A_{1}\right) \geq\left(\frac{2^{k+2}-(-1)^{k+2}}{3}\right)+2^{k}\left(2^{2 k-2}-1\right)$. Therefore, we can move at least $2^{2 k-2}$ pebbles to the vertex $a_{k}$. Now, we have the path $P$ : $a_{k}, a_{k+1}, \ldots, a_{2 k-1}$ of length $k-1$ with at least $2^{2 k-2}$ pebbles. Then by Lemma 2.3 , we can move $2^{k-1}$ pebbles to the vertex $a_{2 k-1}$, which completes the proof.

Lemma 2.8. Let $Z Z_{n}\left(C_{2 k+1}\right)$ be the zig-zag chain graph of $n$ copies of odd cycles. Then the following are true.

1. For $n=2 m, m \geq 1$, we can move at least $2^{k-1}$ pebbles to the vertex $a_{(m-1)(k-1)+m k}$, when $p\left(\left\langle Z Z_{2 m}\left(C_{2 k+1}\right)-A_{2 m}\right\rangle\right) \geq 2^{m(2 k-1)+1}-$ $\left(\frac{2^{k+2}-(-1)^{k+2}}{3}\right)+1$.

2. For $n=2 m+1, m \geq 1$, we can move at least $2^{k-1}$ pebbles to the vertex $a_{m(2 k-1)}$, when $p\left(\left\langle Z Z_{2 m+1}\left(C_{2 k+1}\right)-A_{2 m+1}\right\rangle\right) \geq 2^{(2 m+1) k-m}+$ 


$$
k(2 m+1)-(m+1)-\left(\frac{2^{k+2}-(-1)^{k+2}}{3}\right)+1 .
$$

Proof. We proceed by induction on $m$. For $m=1$, the result follows from Lemma 2.6 and Lemma 2.7. Assume that the result is true for $m^{\prime}<m$. We have to prove the lemma for $m>1$. Consider the graph $Z Z_{n}\left(C_{2 k+1}\right)$ with given number of pebbles according to the choice of $n$. We have to move at least $2^{k-1}$ pebbles to the vertex $a_{(m-1)(k-1)+m k}$, if $n$ is even, otherwise to the vertex $a_{m(2 k-1)}$. We consider the following cases:

Case (1). $n=2 m, m \geq 1$.

Consider the graph $Z Z_{2 m}\left(C_{2 k+1}\right)$ with more than $2^{m(2 k-1)+1}-\left(\frac{2^{k+2}-(-1)^{k+2}}{3}\right)$ pebbles distributed only on the vertices of $\left\langle Z Z_{2 m}\left(C_{2 k+1}\right)-A_{2 m}\right\rangle$. Suppose that the subgraph $S=\left\langle A_{2} \cup A_{3} \cup \ldots \cup A_{2 m-1}\right\rangle$ of $Z Z_{2 m}\left(C_{2 k+1}\right)$ has at least $2^{k(2 m-1)-m+1}+k(2 m-1)-m-\left(\frac{2^{k+2}-(-1)^{k+2}}{3}\right)+1$ pebbles . Then by induction, we can put at least $2^{k-1}$ pebbles to the vertex $a_{(m-1)(k-1)+m k}$. Now, assume that the subgraph $S$ has at most $2^{k(2 m-1)-m+1}+k(2 m-1)-m-$ $\left(\frac{2^{k+2}-(-1)^{k+2}}{3}\right)$ pebbles. We claim the following:

$\operatorname{Claim}(3) \cdot p\left(\left\langle Z Z_{2 m}\left(C_{2 k+1}\right)-A_{2 m}\right\rangle\right)-p(S) \geq\left(\frac{2^{k+2}-(-1)^{k+2}}{3}\right)+2^{k}\left(2^{m(2 k-1)-k}-\right.$ $1)$.

We have,

$$
\begin{aligned}
2^{m(2 k-1)+1}-\left(2^{k(2 m-1)-m+1}+k(2 m\right. & -1)-m)-\left(\frac{2^{k+2}-(-1)^{k+2}}{3}\right)-2^{m(2 k-1)}+2^{k} \\
& \geq 2^{m(2 k-1)}\left(1-\frac{1}{2^{k-1}}\right)-2^{k}-2 m k+k+m \\
& \geq \frac{2^{m k} 2^{m(k-1)}}{2^{k-1}}-2^{k}-2 m k+k+m \\
& >2^{k}\left(2^{m}-1\right)-2 m k+k+m \\
& \geq 2^{k+m-1}-2 m k+k+m \\
& >0, \text { since } 2^{k+m-1} \geq 2 m k .
\end{aligned}
$$

Hence $p\left(A_{1}\right) \geq\left(\frac{2^{k+2}-(-1)^{k+2}}{3}\right)+2^{k}\left(2^{m(2 k-1)-k}-1\right)$. Therefore, we can move at least $2^{m(2 k-1)-k}$ pebbles to the vertex $a_{k}$. Then by using the path $P^{\prime}: a_{k}, a_{k+1}, \ldots, a_{(m-1)(k-1)+m k}$, we can move $2^{k-1}$ pebbles to the vertex 
$a_{(m-1)(k-1)+m k}$. This completes the proof of Case (1).

Case (2). $n=2 m+1, m \geq 1$.

Consider the graph $Z Z_{2 m+1}\left(C_{2 k+1}\right)$ with at least $2^{k(2 m+1)-m}+k(2 m+1)-$ $m-\left(\frac{2^{k+2}-(-1)^{k+2}}{3}\right)$ pebbles distributed only on the vertices of

$\left\langle Z Z_{2 m+1}\left(C_{2 k+1}\right)-A_{2 m+1}\right\rangle$. Suppose that the subgraph

$S^{\prime}=\left\langle A_{2} \cup A_{3} \cup \ldots \cup A_{2 m}\right\rangle$ has more than $2^{m(2 k-1)+1}-\left(\frac{2^{k+2}-(-1)^{k+2}}{3}\right)$ pebbles. Then by induction, we can move at least $2^{k-1}$ pebbles to the vertex $a_{m(2 k-1)}$. Otherwise, we have the following claim to calculate the number of pebbles retained on $A_{1}$.

$\operatorname{Claim}(4) \cdot p\left(\left\langle Z Z_{2 m+1}\left(C_{2 k+1}\right)-A_{2 m+1}\right\rangle\right)-p\left(S^{\prime}\right) \geq\left(\frac{2^{k+2}-(-1)^{k+2}}{3}\right)+2^{k}\left(2^{2 m k-m-1}-\right.$ 1).

We have,

$$
\begin{aligned}
p\left(\left\langle Z Z_{2 m+1}\left(C_{2 k+1}\right)-A_{2 m+1}\right\rangle\right)- & \left(S^{\prime}\right)-\left(\frac{2^{k+2}-(-1)^{k+2}}{3}\right)-2^{k}\left(2^{2 m k-m-1}-1\right) \\
& \geq 2^{2 m k-m}\left(2^{k-1}-2\right)-2^{k}+m(2 k-1)+k \\
& \geq 2^{2 m k-m}-2^{k}+m(2 k-1)+k, \text { for } k \geq 3 \\
& \geq 0, \text { since } 2^{2 m k-m} \geq 2^{k}, k \geq 3 .
\end{aligned}
$$

For $k=2,\left(2^{k-1}-2\right)=0$ and $m(2 k-1)+k \geq 2^{k}$. Thus the claim is true for all $k \geq 2$.

Therefore, we can move at least $2^{2 m k-m-1}$ pebbles to the vertex $a_{k}$, by using the $t$-pebbling number of the odd cycle on $A_{1}$. Now, we can move at least $2^{k-1}$ pebbles to the vertex $a_{m(2 k-1)}$ by using the path $P^{\prime \prime}$ : $a_{k}, a_{k+1}, \ldots, a_{m(2 k-1)}$. This completes the proof of Case (2).

\section{Pebbling number}

In this section, we find the pebbling number of the zig-zag chain graph of $n$ copies of odd cycles.

Theorem 3.1. Let $Z Z_{2}\left(C_{2 k+1}\right)$ be the zig-zag chain graph of two copies of odd cycles. Then for $k \geq 2, f\left(Z Z_{2}\left(C_{2 k+1}\right)\right)=2^{2 k}$. 
Proof. Put $2^{2 k}-1$ pebbles on the vertex $x$. Then we can not reach the vertex $y$, since the diameter of the graph $Z Z_{2}\left(C_{2 k+1}\right)$ is $2 k$. Consider the graph $Z Z_{2}\left(C_{2 k+1}\right)$ with a distribution of at least $2^{2 k}$ pebbles on its vertices. Let $v$ be our specified vertex. Without loss of generality, assume that $v \in A_{2}$. We consider the following cases:

Case (3). $v \in V\left(A_{2}\right)-\{y\}$.

Assume that $p\left(A_{2}\right)<\left(\frac{2^{k+2}-(-1)^{k+2}}{3}\right)-1$. Then more than $2^{2 k}-\left(\frac{2^{k+2}-(-1)^{k+2}}{3}\right)$ pebbles are retained on the vertices of the subgraph $\left\langle Z Z_{2 m}\left(C_{2 k+1}\right)-A_{2}\right\rangle$. By Lemma 2.6 , we can reach any specified vertex except the vertex $y$.

Case (4). $v=y$ is the target vertex.

We have two paths $A: a_{1}, a_{2}, \ldots ., a_{2 k-1}, y$ and $A^{\prime}: b_{1}, b_{2}, \ldots ., z, y$. If $A$ or $A^{\prime}$ has at least $2^{2 k-1}$ pebbles, then we are done. So, we assume that both $A$ and $A^{\prime}$ have at most $2^{2 k-1}-1$ pebbles. Thus, we have the following:

$$
\begin{aligned}
& p(A) \leq 2^{2 k-1}-1 \text { and } \\
& p\left(A^{\prime}\right) \leq 2^{2 k-1}-1 .
\end{aligned}
$$

Then the remaining number of pebbles are retained on the vertex $x$. Now, we apply the pebbling steps and move the pebbles from the vertex $x$ either to the path $A$ or to the path $A^{\prime}$. Then we must have,

$$
\begin{aligned}
& \frac{p(x)}{2}+p(A) \geq 2^{2 k-1} \text { or } \\
& \frac{p(x)}{2}+p\left(A^{\prime}\right) \geq 2^{2 k-1} .
\end{aligned}
$$

Otherwise, $p(x)+p(A)+p\left(A^{\prime}\right)<2^{2 k}$, which is a contradiction to the total number of pebbles distributed on the vertices of the graph.

Theorem 3.2. Let $Z Z_{3}\left(C_{2 k+1}\right)$ be the zig-zag chain graph of three copies of odd cycles. Then for $1<k<6, f\left(Z Z_{3}\left(C_{2 k+1}\right)\right)=2^{3 k-1}+3 k-2$.

Proof. Put $2^{3 k-1}-1$ pebbles on the vertex $x$, and put one pebble on each $b_{i}$ 's, where $1 \leq i \leq 3 k-2$. In this distribution, we cannot reach the vertex $y$. Consider the graph $Z Z_{3}\left(C_{2 k+1}\right)$ with a distribution of at least $2^{3 k-1}+3 k-2$ pebbles on its vertices. We have to move a pebble to any 
arbitrary vertex $v \in A_{p}, 1 \leq p \leq 3$. We consider the following cases:

Case (5). $v \in A_{2}$.

Suppose $p\left(A_{2} \cup A_{3}\right) \geq 2^{2 k}$. Then by Theorem 3.1, we can pebble the vertex $v$. Assume that at most $2^{2 k}-1$ pebbles are distributed on the vertices of the subgraph $A_{2} \cup A_{3}$. Then the number of pebbles distributed on the vertices of $A_{1}$ is at least $2^{2 k}$. Clearly, $p\left(A_{1} \cup A_{2}\right)>2^{2 k}$. Again by Theorem 3.1 , we can move a pebble to the vertex $v$.

Case (6). $v \in A_{1}$ or $A_{3}$.

Without loss of generality, let us assume that $v \in A_{3}$ and $p\left(A_{3}\right) \leq\left(\frac{2^{k+2}-(-1)^{k+2}}{3}\right)-1$. Then the remaining number of pebbles distributed only on the vertices of the subgraph $A_{1} \cup A_{2}$ is at least $2^{3 k-1}+$ $3 k-1-\left(\frac{2^{k+2}-(-1)^{k+2}}{3}\right)$. Let $v \in V\left(A_{3}\right)-\{y, z\}$. Then by Lemma 2.7, we can easily reach the vertex $v$. Now, assume that $v \in\{y, z\}$. Without loss of generality, take $v=y$. We have two paths $B: a_{k}, a_{k+1}, \ldots, a_{3 k-2}, y$ and $B^{\prime}: b_{k+1}, b_{k+2}, \ldots, z, y$ each of length $2 k-1$. Suppose $p(B) \geq 2^{2 k-1}$ or $p\left(B^{\prime}\right) \geq 2^{2 k-1}$. Then we are done. Therefore, assume that, $p(B) \leq 2^{2 k-1}-1$ and $p\left(B^{\prime}\right) \leq 2^{2 k-1}-1$. Without loss of generality, assume that all the pebbles are distributed on $A_{1}$. We have the following claim to pebble the vertex $y$.

$\operatorname{Claim}(5) .2^{3 k-1}+3 k-2 \geq\left(\frac{2^{k+2}-(-1)^{k+2}}{3}\right)+2^{k}\left(2^{2 k-1}-1\right)$.

We have,

$$
\begin{array}{r}
2^{3 k-1}+3 k-2-\left(\frac{2^{k+2}-(-1)^{k+2}}{3}\right)-2^{k}\left(2^{2 k-1}-1\right) \\
=3 k+2^{k}-\left(\frac{2^{k+2}-(-1)^{k+2}}{3}\right)-2 .
\end{array}
$$

For all $k<6$,

$$
2^{k}+3 k \geq\left(\frac{2^{k+2}-(-1)^{k+2}}{3}\right)+2 .
$$

Hence $p\left(A_{1}\right) \geq\left(\frac{2^{k+2}-(-1)^{k+2}}{3}\right)+2^{k}\left(2^{2 k-1}-1\right)$. Now, we can move $2^{2 k-1}$ pebbles to the vertex $a_{k}$, and then we move a pebble to the vertex $y$. 
Theorem 3.3. Let $Z Z_{n}\left(C_{2 k+1}\right)$ be the zig-zag chain graph of $n$ copies of odd cycles. Then the following are true.

1. For $n=2 m, m \geq 1$ and $1<k<6$,

$$
f\left(Z Z_{2 m}\left(C_{2 k+1}\right)\right)=2^{m(2 k-1)+1} .
$$

2. For $n=2 m+1, m \geq 1$ and $1<k<6$,

$$
f\left(Z Z_{2 m+1}\left(C_{2 k+1}\right)\right)=2^{(2 m+1) k-m}+(2 m+1) k-(m+1) .
$$

Proof. For $n=2 m, m \geq 1$, by placing $2^{m(2 k-1)+1}-1$ pebbles on the vertex $x$, we cannot reach the vertex $y$. For $n=2 m+1 m \geq 1$, by placing $2^{(2 m+1) k-m}-1$ pebbles on the vertex $x$ and one pebble on each $b_{i}$, we cannot reach the vertex $y$.

We proceed by induction on $m$. For $m=1$, the result follows from Theorem 3.1 and Theorem 3.2. Assume that the result is true for $m^{\prime}<m$. Let $v \in A_{p}, 1 \leq p \leq n$. We consider the following cases:

Case (7). $n$ is even. ie., $n=2 m, m \geq 2$.

Consider the graph $Z Z_{2 m}\left(C_{2 k+1}\right)$ with a distribution of at least $2^{m(2 k-1)+1}$ pebbles on its vertices. We consider the following subcases:

Subcase $7(a) \cdot p$ is odd and $1<p<n$.

Let $n=2 m$, and $m \geq 2$. Then the graph $Z Z_{2 m}\left(C_{2 k+1}\right)$ can be partitioned into two subgraphs say $M_{1}$ and $M_{2}$, where $M_{1} \cong Z Z_{p}\left(C_{2 k+1}\right)$, $M_{2} \cong Z Z_{s}\left(C_{2 k+1}\right)$ and $2 m=p+s-1$. Put $p=2 p^{\prime}+1$, and $s=2 s^{\prime}$. Clearly, $v \in A_{p} \subseteq M_{1} \cap M_{2}$. Suppose $p\left(M_{1}\right) \geq 2^{\left(2 p^{\prime}+1\right) k-p^{\prime}}+\left(2 p^{\prime}+1\right) k-\left(p^{\prime}+1\right)$. Then we are done. Suppose $p\left(M_{2}\right)<2^{\left(2 p^{\prime}+1\right) k-p^{\prime}}+\left(2 p^{\prime}+1\right) k-\left(p^{\prime}+1\right)$. Now, we claim the following:

$\operatorname{Claim}(6) \cdot p\left(Z Z_{2 m}\left(C_{2 k+1}\right)\right)-p\left(M_{1}\right) \geq 2^{s^{\prime}(2 k-1)+1}$.

Now, $p\left(Z Z_{2 m}\left(C_{2 k+1}\right)\right)-p\left(M_{1}\right)-2^{s^{\prime}(2 k-1)+1}$

$=2^{2 p^{\prime} k-p^{\prime}}\left(2^{2 s^{\prime} k-s^{\prime}}-2^{k}\right)+2^{2 s^{\prime} k-s^{\prime}}\left(2^{2 p^{\prime} k-p^{\prime}}-2\right)-\left(2 p^{\prime}+1\right) k+p^{\prime}$ 


$$
\begin{aligned}
& >2^{2 p^{\prime} k-p^{\prime}}+2^{2 s^{\prime} k-s^{\prime}}-2^{p^{\prime} k+1} \\
& >2^{2 s^{\prime} k-s^{\prime}} \\
& >0
\end{aligned}
$$

This implies that $p\left(M_{2}\right) \geq 2^{s^{\prime}(2 k-1)+1}$. Therefore, we can reach the vertex $v$.

Subcase $7(b) . p$ is even and $1<p<n$.

Put $p=2 p^{\prime}$. Then we get that $s=2 s^{\prime}+1$. Suppose $p\left(M_{1}\right) \geq 2^{p^{\prime}(2 k-1)+1}$. Then we can pebble the target. Therefore, assume that $p\left(M_{1}\right) \leq 2^{p^{\prime}(2 k-1)+1}-$ 1.

$\operatorname{Claim}(7) \cdot p\left(Z Z_{2 m}\left(C_{2 k+1}\right)\right)-p\left(M_{1}\right) \geq 2^{\left(2 s^{\prime}+1\right) k-s^{\prime}}+\left(2 s^{\prime}+1\right) k-s^{\prime}$.

The reader can easily verify that the claim follows from Claim (6).

Subcase $7(c) \cdot p=1$ or $p=n$.

Without loss of generality, assume that $v \in A_{n}$ and $p\left(A_{n}\right) \leq\left(\frac{2^{k+2}-(-1)^{k+2}}{3}\right)-$ 1. Let $v \in V\left(A_{n}\right)-\{y\}$. Then by Lemma 2.8 , we can easily pebble the target. Take $v=y$. We have two paths $C: a_{1}, a_{2}, \ldots, y$ and $C^{\prime}: b_{1}, b_{2}, \ldots, y$. If $C$ or $C^{\prime}$ has at least $2^{m(2 k-1)}$ pebbles, then we can reach the target. Therefore assume that,

$$
\begin{aligned}
& p(C) \leq 2^{m(2 k-1)}-1 \text { and } \\
& p\left(C^{\prime}\right) \leq 2^{m(2 k-1)}-1
\end{aligned}
$$

Then the remaining pebbles are retained on the vertex $x$. Now, we apply the pebbling steps and move the pebbles from the vertex $x$ to the path $C$ or to the path $C^{\prime}$. We must have,

$$
\begin{aligned}
& \frac{p(x)}{2}+p(C) \geq 2^{m(2 k-1)} \text { or } \\
& \frac{p(x)}{2}+p\left(C^{\prime}\right) \geq 2^{m(2 k-1)} .
\end{aligned}
$$

Otherwise, $p(x)+p(C)+p\left(C^{\prime}\right)<2^{m(2 k-1)+1}$, which is a contradiction to the total number of pebbles distributed on the vertices of the graph. 
Case (8). $n$ is odd. ie., $n=2 m+1, m \geq 2$.

Let $n=2 m+1$. Consider the graph $Z Z_{2 m+1}\left(C_{2 k+1}\right)$ with a distribution of at least $2^{(2 m+1) k-m}+(2 m+1) k-(m+1)$ pebbles on its vertices. Let $v \in A_{p}, 1 \leq p \leq n$. We partition the graph $Z Z_{2 m+1}\left(C_{2 k+1}\right)$ into two subgraphs, say $M_{1} \cong Z Z_{p}\left(C_{2 k+1}\right)$, and $M_{2} \cong Z Z_{s}\left(C_{2 k+1}\right)$. Clearly, $M_{1} \cap M_{2} \cong A_{p}$. We consider the following subcases to pebble the target.

Subcase $8(a) \cdot p$ is odd and $1<p<n$.

Put $2 m+1=\left(2 p^{\prime}+1\right)+\left(2 s^{\prime}+1\right)-1$. Then we get that $m=p^{\prime}+s^{\prime}$. Here $p=$ $2 p^{\prime}+1$ and $s=2 s^{\prime}+1$. Suppose $p\left(M_{1}\right) \leq 2^{\left(2 p^{\prime}+1\right) k-p^{\prime}}+\left(2 p^{\prime}+1\right) k-\left(p^{\prime}+1\right)-1$. We claim the following:

$\operatorname{Claim}(8) \cdot p\left(Z Z_{2 m+1}\left(C_{2 k+1}\right)\right)-p\left(M_{1}\right) \geq 2^{\left(2 s^{\prime}+1\right) k-s^{\prime}}+\left(2 s^{\prime}+1\right) k-\left(s^{\prime}+1\right)$.

Now,

$$
\begin{aligned}
& 2^{(2 m+1) k-m}-2^{\left(2 p^{\prime}+1\right) k-p^{\prime}}-2^{\left(2 s^{\prime}+1\right) k-s^{\prime}}+(2 m+1) k-\left(2 p^{\prime}+1\right) k-\left(2 s^{\prime}+1\right) k \\
&=2^{\left(2 p^{\prime}+2 s^{\prime}+1\right) k-p^{\prime}-s^{\prime}}-2^{\left(2 p^{\prime}+1\right) k-p^{\prime}}-2^{\left(2 s^{\prime}+1\right) k-s^{\prime}}-k \\
&=2^{k}\left(2^{p^{\prime}(2 k-1)} \cdot 2^{s^{\prime}(2 k-1)}-2^{p^{\prime}(2 k-1)}-2^{s^{\prime}(2 k-1)}\right)-k \\
&>2^{k}-k \\
&>0, \text { since, } 2^{p^{\prime}(2 k-1)} \cdot 2^{s^{\prime}(2 k-1)}-2^{p^{\prime}(2 k-1)}-2^{s^{\prime}(2 k-1)}>0 .
\end{aligned}
$$

Hence $p\left(M_{2}\right) \geq 2^{\left(2 s^{\prime}+1\right) k-s^{\prime}}+\left(2 s^{\prime}+1\right) k-\left(s^{\prime}+1\right)$. Therefore, we can reach the vertex $v$ by induction.

Subcase $8(b) . p$ is even and $1<p<n$.

Put $2 m+1=2 p^{\prime}+2 s^{\prime}-1$. Here $p=2 p^{\prime}$ and $s=2 s^{\prime}$. Suppose $p\left(M_{1}\right) \geq 2^{p^{\prime}(2 k-1)+1}$. Then we can reach the vertex $v$. Therefore assume that $p\left(M_{1}\right) \leq 2^{p^{\prime}(2 k-1)+1}-1$. We claim the following:

$\operatorname{Claim}(9) \cdot p\left(Z Z_{2 m+1}\left(C_{2 k+1}\right)\right)-p\left(M_{1}\right) \geq 2^{s^{\prime}(2 k-1)+1}$.

We have, 


$$
\begin{aligned}
2^{\left(2 p^{\prime}+2 s^{\prime}-1\right) k-\left(p^{\prime}+s^{\prime}-1\right)} & -2^{p^{\prime}(2 k-1)+1}-2^{s^{\prime}(2 k-1)+1}+m(2 k-1)+k-1 \\
& >2^{2 p^{\prime} k-p^{\prime}}\left(2^{2 s^{\prime} k-s^{\prime}-k}-2\right)+2^{2 s^{\prime} k-s^{\prime}}\left(2^{2 p^{\prime} k-p^{\prime}-k}-2\right) \\
& >2^{2 p^{\prime} k-p^{\prime}}\left(2^{s^{\prime}}-2\right)+2^{2 s^{\prime} k-s^{\prime}}\left(2^{p^{\prime}}-2\right) \\
& \geq 0 .
\end{aligned}
$$

Hence, $p\left(M_{2}\right) \geq 2^{s^{\prime}(2 k-1)+1}$, so we are done.

Subcase $8(c) . v \in A_{p}$ and $p=1$ or $p=n$.

Let us assume that, $v \in A_{n}$. Let $v \in V\left(A_{p}\right)-\{y, z\}$. Then by Lemma 2.8 , we can easily reach the vertex $v$. Let $v \in\{y, z\}$. Without loss of generality, assume that $v=y$. We have two paths $D: a_{k}, a_{k+1}, \ldots, y$ and $D^{\prime}: b_{k+1}, b_{k+2}, \ldots, z, y$ each of length $(2 k-1) m$. Suppose $p(D) \geq 2^{(2 k-1) m}$ or $p\left(D^{\prime}\right) \geq 2^{(2 k-1) m}$. Then we can pebble the vertex $y$. So assume that $p(D) \leq 2^{(2 k-1) m}-1$ and $p\left(D^{\prime}\right) \leq 2^{(2 k-1) m}-1$. Without loss of generality, assume that all the pebbles are distributed on $A_{1}$.

$\operatorname{Claim}(10) \cdot p\left(A_{1}\right) \geq\left(\frac{2^{k+2}-(-1)^{k+2}}{3}\right)+2^{k}\left(2^{m(2 k-1)}-1\right)$.

Now we have,

$$
\begin{array}{r}
2^{(2 m+1) k-m}+(2 m+1) k-( \\
m+1)-\left(\frac{2^{k+2}-(-1)^{k+2}}{3}\right)-2^{k}\left(2^{m(2 k-1)}-1\right) \\
=2^{k}+(2 m+1) k-\left(\frac{2^{k+2}-(-1)^{k+2}}{3}\right)-(m+1) .
\end{array}
$$

We can easily verify that for all $k<6$,

$$
2^{k}+(2 m+1) k \geq\left(\frac{2^{k+2}-(-1)^{k+2}}{3}\right)-(m+1) .
$$

Hence, we can move a pebble to the vertex $y$.

\section{4. t- Pebbling Number}

In this section, we determine the t-pebbling number of zig-zag chain graph of $n$ copies of odd cycles. 
Theorem 4.1. Let $Z Z_{n}\left(C_{2 k+1}\right), k \geq 2$ be the zig-zag chain graph of $n$ copies of odd cycles.

1. For $n=2 m, m \geq 1$, we have, $f_{t}\left(Z Z_{2 m}\left(C_{2 k+1}\right)\right)=t .2^{m(2 k-1)+1}$.

2. For $n=2 m+1, m \geq 1$, we have, $f_{t}\left(Z Z_{2 m+1}\left(C_{2 k+1}\right)\right)=t .2^{(2 m+1) k-m}+$ $(2 m+1) k-(m+1)$.

Proof. 1. Place $t .2^{m(2 k-1)+1}-1$ pebbles on the vertex $x$, we cannot put $t$ pebbles on the vertex $y$.

Consider the graph $Z Z_{2 m}\left(C_{2 k+1}\right)$ with a distribution of at least $t .2^{m(2 k-1)+1}$ pebbles on its vertices. We prove this theorem by induction on $t$. For $t=1$, the result follows from the Theorem 3.3. Assume that the result is true for $t^{\prime}<t$. Let $v \in A_{p}, 1 \leq p \leq 2 m$, be any target vertex. We have to move $t$ pebbles to the vertex $v$. Consider the following cases:

Case $(9) \cdot p(v)=0$.

We can move one pebble to the vertex $v$ at a cost of at most $2^{m(2 k-1)+1}$ pebbles. Then the number of pebbles remaining in $Z Z_{2 m}\left(C_{2 k+1}\right)$ is at least $(t-1) 2^{m(2 k-1)+1}$. Thus by induction, we can move $t-1$ additional pebbles to $v$.

Case (10). $p(v)=l, 1 \leq l \leq t-1$ and $t \geq 2$.

We have to move $t-l$ additional pebbles to the vertex $v$.

Now, $f_{t}\left(Z Z_{2 m}\left(C_{2 k+1}\right)\right)-l=t .2^{m(2 k-1)+1}-l$

$$
\begin{aligned}
& \geq(t-l+l) 2^{m(2 k-1)+1}-l \\
& =(t-l) 2^{m(2 k-1)+1}+l 2^{m(2 k-1)+1}-l \\
& >(t-l) 2^{m(2 k-1)+1} \\
& =f_{t-l}\left(Z Z_{2 m}\right) .
\end{aligned}
$$

By induction, we can move $t-l$ additional pebbles to the vertex $v$.

2. Place $t .2^{(2 m+1) k-m}-1$ pebbles on the vertex $x$ and put one pebble on each $b_{i}$. We cannot move $t$ pebbles to the vertex $y$. 
Consider the graph $Z Z_{2 m+1}\left(C_{2 k+1}\right)$ with a distribution of at least $t .2^{(2 m+1) k-m}+$ $(2 m+1) k-(m+1)$ pebbles on its vertices. We prove this theorem by induction on $t$. Let $v \in A_{p}, 1 \leq p \leq 2 m+1$. We have the following cases:

Case $(11) \cdot p(v)=0$.

The graph $Z Z_{2 m+1}\left(C_{2 k+1}\right)$ can be partitioned into two subgraphs say, $M_{1} \cong Z Z_{p}\left(C_{2 k+1}\right)$ and $M_{2} \cong Z Z_{s}\left(C_{2 k+1}\right)$, where $M_{1} \cap M_{2} \cong A_{p}$.

Subcase 11(a). $v \in A_{p}, p$ is even and $1<p<2 m+1$.

Put $2 m+1=2 p^{\prime}+2 s^{\prime}-1$, where $p=2 p^{\prime}$ and $s=2 s^{\prime}$. Suppose $p\left(M_{1}\right) \geq t .2^{p^{\prime}(2 k-1)+1}$. Then we are done. Therefore assume that $p\left(M_{1}\right) \leq$ $t .2^{p^{\prime}(2 k-1)+1}-1$. We claim the following:

$\operatorname{Claim}(11) \cdot p\left(Z Z_{2 m+1}\left(C_{2 k+1}\right)\right)-p\left(M_{1}\right) \geq t .2^{s^{\prime}(2 k-1)+s^{\prime}+1}$.

We have,

$$
\begin{aligned}
t .2^{(2 m+1) k-m}+ & (2 m+1) k-(m+1)-\left(t .2^{p^{\prime}(2 k-1)+1}-1\right)-t .2^{s^{\prime}(2 k-1)+1} \\
& >t .2^{2 p^{\prime} k-p^{\prime}}\left(2^{\left(2 s^{\prime}-1\right) k-s^{\prime}}-2\right)+t .2^{2 s^{\prime} k-s^{\prime}}\left(2^{\left(2 p^{\prime}-1\right) k-p^{\prime}}-2\right) \\
& >0, \text { since } s^{\prime} \geq 1 \text { and } p^{\prime} \geq 1
\end{aligned}
$$

Hence, $P\left(Z Z_{s}\left(C_{2 k+1}\right)\right) \geq t \cdot 2^{s^{\prime}(2 k-1)+s^{\prime}+1}$. Therefore, we can pebble the vertex $v$.

Subcase 11(b). $v \in A_{p}, p$ is odd, and $1<p<2 m+1, m \geq 1$.

Put $2 m+1=\left(2 p^{\prime}+1\right)+\left(2 s^{\prime}+1\right)-1$, where $p=2 p^{\prime}+1$ and $s=2 s^{\prime}+1$. Suppose $P\left(M_{1}\right) \geq t .2^{\left(2 p^{\prime}+1\right) k-p^{\prime}}+\left(2 p^{\prime}+1\right) k-\left(p^{\prime}+1\right)$. Then we are done. Now, assume that $P\left(M_{1}\right) \leq t .2^{\left(2 p^{\prime}+1\right) k-p^{\prime}}+\left(2 p^{\prime}+1\right) k-\left(p^{\prime}+1\right)-1$. We claim the following:

$\operatorname{Claim}(12) \cdot p\left(Z Z_{2 m+1}\left(C_{2 k+1}\right)\right)-p\left(M_{1}\right) \geq t \cdot 2^{\left(2 s^{\prime}+1\right) k-s^{\prime}}+\left(2 s^{\prime}+1\right) k-\left(s^{\prime}+1\right)$.

We have,

$$
\begin{array}{r}
p\left(Z Z_{2 m+1}\left(C_{2 k+1}\right)\right)-p\left(M_{1}\right)-t .2^{\left(2 s^{\prime}+1\right) k-s^{\prime}}-\left(2 s^{\prime}+1\right) k+\left(s^{\prime}+1\right) \\
>t .2^{\left(2 p^{\prime}+2 s^{\prime}+1\right) k-p^{\prime}-s^{\prime}}-t .2^{\left(2 p^{\prime}+1\right) k-p^{\prime}}-t .2^{\left(2 s^{\prime}+1\right) k-s^{\prime}}
\end{array}
$$




$$
\begin{aligned}
& =t .2^{k}\left(2^{2 p^{\prime} k+2 s^{\prime} k-p^{\prime}-s^{\prime}}-2^{2 p^{\prime} k-p^{\prime}}-2^{2 s^{\prime} k-s^{\prime}}\right) \\
& =t .2^{k}\left(2^{2 p^{\prime} k-p^{\prime}} \cdot 2^{2 s^{\prime} k-s^{\prime}}-2^{2 p^{\prime} k-p^{\prime}}-2^{2 s^{\prime} k-s^{\prime}}\right) \\
& >0 .
\end{aligned}
$$

Hence, $p\left(Z Z_{s}\left(C_{2 k+1}\right)\right) \geq t \cdot 2^{\left(2 s^{\prime}+1\right) k-s^{\prime}}+\left(2 s^{\prime}+1\right) k-\left(s^{\prime}+1\right)$. Therefore, we can pebble the vertex $v \in A_{p}$.

Subcase $11(c) . v \in A_{p}$, and $p=1$ or $p=2 m+1$.

Without loss of generality, assume that $v \in A_{2 m+1}$. Let $v=y$. We have two paths $E: x, a_{1}, \ldots, y$ and $E^{\prime}: b_{1}, b_{2}, \ldots, z, y$ each of length $(2 m+1) k-m$. Suppose that the path $E$ has at least $2^{(2 m+1) k-m}$ pebbles. We can move one pebble to the vertex $y$. Then the number of pebbles in $Z Z_{2 m+1}\left(C_{2 k+1}\right)$ is at least $(t-1) 2^{(2 m+1) k-m}+(2 m+1) k-(m+1)$. Now by induction, we can move $t-1$ additional pebbles to the vertex $y$. Suppose $p(E) \leq 2^{(2 m+1) k-m}-1$. The number of pebbles in $E^{\prime}$ is at least $(t-1) 2^{(2 m+1) k-m}+(2 m+1) k-(m+$ $1)$. By using exactly $(t-1) 2^{(2 m+1) k-m}$ pebbles, we can move $t-1$ pebbles to the vertex $y$. Also the number of pebbles unused in $Z Z_{2 m+1}\left(C_{2 k+1}\right)$ is at least $2^{(2 m+1) k-m}+(2 m+1) k-(m+1)$. By Theorem 3.3, we can move an additional pebble to the target vertex.

Case (12). $p(v)=l, 1 \leq l \leq t-1$.

We have to move $t-l$ additional pebbles to the vertex $v \in A_{p}, 1 \leq p \leq 2 m+$ 1. Clearly, $p\left(Z Z_{2 m+1}\left(C_{2 k+1}\right)\right)-l \geq(t-l) 2^{(2 m+1) k-m}+(2 m+1) k-(m+1)$. Therefore, we can move $t-l$ additional pebbles to the target vertex $v$.

\section{References}

[1] G. Chartrand and L. Lesniak, Graphs \& digraphs, 4th ed. Boca Raton, FL: CRC Press, 2004.

[2] F. Chung, "Pebbling in hypercubes", SIAM Journal on Discrete Mathematics, vol. 2, no. 4, pp. 467-472, Nov. 1989, doi:10.1137/0402041.

[3] P. Lemke and D. Kleitman, "An addition theorem on the integers modulo n", Journal of Number Theory, vol. 31, no. 3, pp. 335-345, Mar. 1989, doi: 10.1016/0022-314X(89)90077-2. 
[4] D. Moews, "Pebbling graphs", Journal of Combinatorial Theory, Series $B$, vol. 55, no. 2, pp. 244-252, 1992, doi: 10.1016/00958956(92)90043-W.

[5] L. Pachter, H. Snevily, and B. Voxman, "On pebbling graphs", Congressus Numerantium, vol. 107, pp. 65-80, 1995.

[6] D. Herscovici and A. Higgins, "The pebbling number of C5 × C5", Discrete Mathematics, vol. 187, no. 1-3, pp. 123-135, Jun. 1998, doi: 10.1016/S0012-365X(97)00229-X.

[7] G. Hurlbert, The Graph Pebbling Page. [Online]. Available: http://mingus.la.asu.edu/ hurlbert/pebbling/pebb.html

[8] C. Cusack et al., Algoraph. [Online]. Available: http://algoraph.hope.edu

[9] D. Herscovici, "Grahams pebbling conjecture on products of cycles", Journal of Graph Theory, vol. 42, no. 2, pp. 141-154, Jan. 2003, doi: 10.1002/jgt.10080.

[10] A. Lourdusamy, "t-pebbling the graphs of diameter two", Acta Ciencia Indica, Mathematics, vol.29, no. 3, pp. 465-470, 2003.

[11] A. Lourdusamy, "t-pebbling the product of graphs", Acta Ciencia Indica, Mathematics, vol. 32, no. 1, pp. 171-176, 2006.

[12] A. Lourdusamy and A. Tharani, "On t-pebbling graphs", Utilitas Mathematica, vol. 87, pp. 331-342, 2012.

[13] A. Lourdusamy, S. Jeyaseelan and A. Tharani, "t-pebbling the product of fan graphs and the product of wheel graphs", International Mathematical Forum, vol. 4, no. 32, pp. 1573-1585, 2009. [On line]. Available: http://bit.ly/2YZI2QA

[14] A. Lourdusamy and J. Steffi, "The pebbling number of zig-zag chain graph of even cycles", Sciencia Acta Xaveriana, vol. 8, no. 1, pp.49-62, 2017.

[15] A. Lourdusamy and J. Steffi, "The 2t-Pebbling property on the zig-zag chain graph of $\mathrm{n}$ odd cycles", International Journal of pure and applied mathematics, vol. 117, special issue, no. 14, pp. 200-218, 2017. [On line], Available: http://bit.ly/2MT0LLq 